\title{
Magnetic Fluxgate Sensor Characteristics Modeling Using Extended Preisach Model
}

\author{
P. Frydrych*, R. SzeWCZYK AND J. SAlaCH \\ Institute of Metrology and Biomedical Engineering, Warsaw University of Technology, ul. A. Boboli 8, 02-525 \\ Warszawa, Poland
}

\begin{abstract}
This paper presents the implementation of the extended Preisach model for fluxgate sensor characteristics modeling. The developed model enables the optimization of fluxgate sensor parameters for a given core material. Model consists of two parts. The first one describes magnetic characteristics of the core, while the second part calculates output signal according to sensor dimensions and electric circuit parameters. The model is easy to adapt for modeling of other devices with magnetic cores. It can be used instead of costly and time consuming prototype testing.
\end{abstract}

DOI: $10.12693 /$ APhysPolA.126.18

PACS: 75.60.-d, 07.07.Df

\section{Introduction}

It is widely known that fluxgate sensors are cheap, robust and sensitive, compared to other magnetic field sensor types. Their parameters are strongly determined by parameters and magnetic characteristics of the core material. Fluxgate sensors parameters are hardly predictable due to the nonlinear material characteristics and its dimension and frequency dependence. This in turn demands prototype testing. Furthermore, in digital power conversion devices unsymmetrical signals in magnetic cores are used. Coercivity, saturation and core losses calculated by manufacturer are not enough to predict device operating characteristic. Application of amorphous ribbons for cores of fluxgate sensors [1] and other electronic sensors [2] increases.

Presented model is able to predict core response for every signal given. It enables easy optimization of fluxgate sensors and digital power conversion devices using amorphous ribbon cores.

\section{Construction of the sensor}

In this research the fluxgate sensor in Foerster configuration was tested. Single layer frame shaped sensor core was made of as-quenched $\mathrm{Fe}_{80} \mathrm{~B}_{11} \mathrm{Si}_{9}$ amorphous ribbon [3]. The inner diameter of the frame was $25 \mathrm{~mm}$ and outer $35 \mathrm{~mm}$. Each branch of frame shaped core had 25 sensing and 15 excitation turns. Coils configuration in sensing circuit was changeable, so it was possible to measure average magnetic hysteresis loops of the core placed in the fluxgate sensor.

\section{Modeling method}

Model consists of two parts. The first one is Preisach model of magnetic characteristics of the core. Model parameters are optimised for representative static hysteresis loops, which are connected only with material properties.

\footnotetext{
*corresponding author; e-mail: p.frydrych@mchtr.pw.edu.pl
}

Second part is connected with core dimensions, coils and excitation parameters. This part also takes into account losses in the material. This approach was chosen to simplify material testing process before modeling.

\section{Magnetic characteristics model}

Preisach based model is the most suitable way for modeling magnetic characteristics of the amorphous materials. Due to statistical nature, this model correlates with the microstructural disorder characteristics for that kind of materials [4]. Preisach plane can also be used to record material magnetisation for every moment of magnetisation process, what is important for calculation of its behaviour for nonharmonic signals.

The Preisach plane is a region between increasing and decreasing axis. It represents the distribution of coercivity operators in a material. Operators can switch up and down. For increasing field all operators which have lower $\alpha$ value than the actual field value switch up. Whereas for decreasing field all operators with higher $\beta$ value switch down. In this model operators matrix represents the Preisach plane. Two-dimensional normal distribution was assumed (Fig. 2). Center of distribution has to be on the symmetry axis, because of symmetrical coercivity of material. As a result there are only four distribution parameters: $A$ - scaling factor, $m$ - mean value of domain coercivity field, $s_{c}$ - coercivity variance and $s_{i}$-interaction variance.

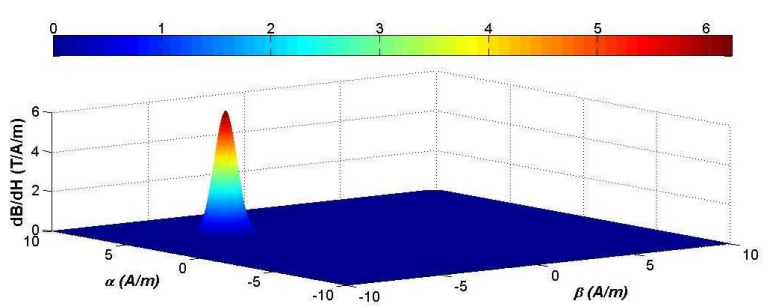

Fig. 1. Preisach plane with distribution of domain coercivity. 


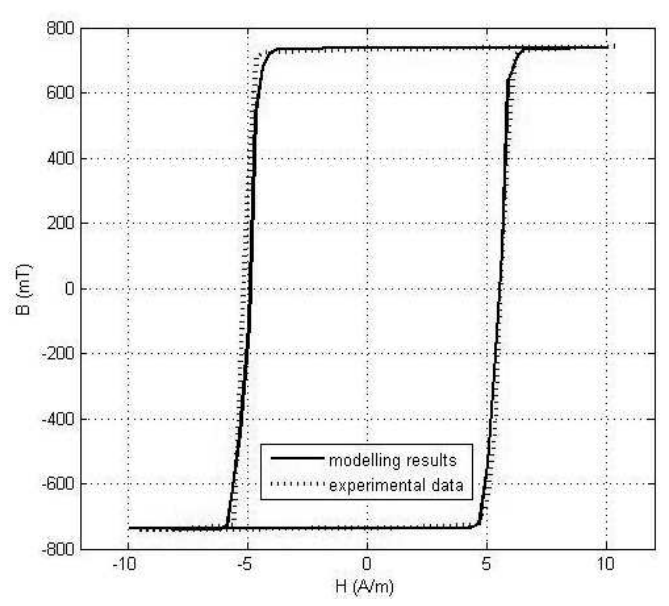

Fig. 2. Results of modeling for quasi-static hysteresis loop for as-quenched $\mathrm{Fe}_{80} \mathrm{~B}_{11} \mathrm{Si}_{9}$.

Model parameters were optimised for static loops measured for multilayer rolled ribbon instead of single layer frame shaped core, which was used in fluxgate sensor. The reason was too weak signal for frequency lower than $1 \mathrm{~Hz}$. The evolution strategy $(\mu+\lambda)$ was used [5] in the optimisation process. The results of the modeling are shown in Figure 3. The achieved value of $R^{2}$ Pearson coefficient was over 0.98 .

The coercivity field distribution does not change with frequency. To model losses, effective field $H_{e}$ is calculated. Losses are proportional to change of internal magnetisation in time, which is similar to magnetic permeability. It takes into account the dimensions of the core, material structure and conductivity. To calculate $H_{e}$, the Preisach plane was used. The external field moves on surface proportional to distribution of magnetic permeability, and the effective field is a projection of it on the horizontal plane.

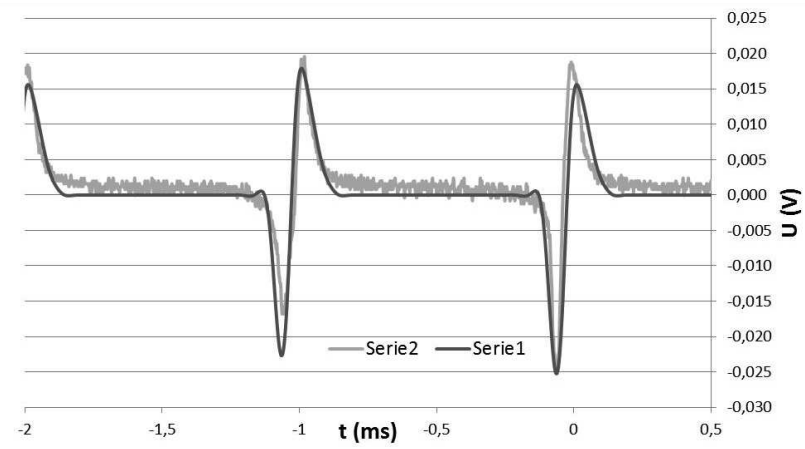

Fig. 3. Results of modeling of fluxgate sensor with asquenched $\mathrm{Fe}_{80} \mathrm{~B}_{11} \mathrm{Si}_{9}$ core.

\section{Sensor model}

The magnetic rate dependent model with the ability to approximate losses in the core is necessary to predict the induction in the core for a given magnetic field. This is especially important, because fluxgate sensors work for frequencies higher than $1 \mathrm{kHz}$. Magnetic field has to be calculated for windings and core dimensions. The output signal can be calculated in the same way. In the Foerster configuration, the signals from two cores are added in electric circuit. Therefore the predicted fluxgate output signal is achieved, which is presented in Figure 5. In this case it can be observed, that there are some imperfections, like oscilloscope offset, influence of filtration, noise and other, which are hard to predict. It can raise doubts, that frame shaped cores could have different characteristics for longitudinal and perpendicular directions due to amorphous ribbon anisotropy. In fact, in this case the induction is averaged, which was proven in research [6].

\section{Conclusions}

Presented model, based on Preisach model and simplified approach to losses prediction, is very suitable for optimisation of fluxgate sensor parameters. It has to be noticed, that model parameters were optimised for characteristics of multilayer rolled core. Therefore, the implementation of that method is very easy. There is also possibility to generalise the model optimised for particular material, for prediction of characteristics of different devices with different shapes of cores made out of that material.

\section{Acknowledgments}

Calculations for the modeling were made in the Interdisciplinary Centre for Mathematical and Computational modeling of Warsaw University, within grant G36-10.

\section{References}

[1] C. Lei, J. Lei, Z. Yang, Y. Zhou, Microsyst. Technol. 19, 167 (2013).

[2] Z. Jitao, L. Ping, W. Yumei, H. Wei, Y. Aichao, Rev. Sci. Instrum. 83, 115001 (2012).

[3] Z. Stoklosa, J. Rasek, P. Kwapulinski, G. Badura, G. Haneczok, L. Pająk, J. Lelątko, A. Kolano-Burian, J. Alloys. Compd 509, 9050 (2011).

[4] P. Andrei, Al. Stancu, O. Caltun, J. Appl. Phys. 83, 6359 (1998).

[5] R. Szewczyk, J. Phys. D: Appl. Phys 40, 4109 (2007).

[6] P. Frydrych, R. Szewczyk, J. Salach, K. Trzcinka, Trans. Magn. 48, 1485 (2012). 